\title{
Wandel der Controllership
}

\section{Liebe Leser,}

wohl über keinen anderen betriebswirtschaftlichen Aufgabenträger sind so viele beschreibende Bilder im Umlauf wie für den Controller. Das bekannteste - und wenig schmeichelnde - von diesen ist ohne Frage das des Erbsenzählers. Es adressiert den großen Umfang von Informationsaufgaben, die Controller leisten. Controller sammeln Zahlen und bereiten sie für das Management auf; sie stehen für die Richtigkeit der Zahlen ein. Controller müssen sich um deren aktuelle Bereitstellung kümmern. Ihnen obliegt das möglichst fehlerfreie Berichtswesen. SAP ist ihnen kein Fremdwort, sondern unverzichtbares Arbeitsmittel.

Eine Vielzahl anderer Bilder beschreibt unterschiedliche Facetten des Zusammenwirkens von Controllern und Managern. Der betriebswirtschaftliche Begleiter und der Lotse bzw. Steuermann betonen eine Ergänzungsfunktion: Controller nehmen ihren Managern bestimmte betriebswirtschaftliche Aufgaben ab, die diese aus Know-how- und/oder Zeitgründen nicht selbst wahrnehmen können oder wollen. Auch der Spürhund und der Kontrolleur ergänzen Manager, allerdings ist die Konnotation dieser Bilder schon eher etwas problematisch: Manager sind nicht rigoros genug, Fehler und Unwirtschaftlichkeiten aufzudecken und bedürfen der Fremdkontrolle. Der Bremser geht in dieselbe Richtung. Manager werden hiermit als notorisch überoptimistisch gesehen; ihre hochfliegenden Vorstellungen bedürfen der realistischen Korrektur durch den Controller, sie sind durch diesen auf den Boden der Tatsachen zurückzuholen. Das ökonomische Gewissen richtet sich mehr auf die Wollensseite der Manager. Hinter ihm steht die latente Gefahr, dass Manager eher an sich (Karriere, Macht, Prestige) als an das Unternehmen denken. Der Hofnarr schließlich zeigt den Weg, wie Controller ihre begrenzende Funktion gegenüber dem Management in geeigneter Weise spielen können: direkte Konfrontation ist gefährlich, Geschick, die richtigen Worte zu finden, unabdingbar.

Controllerbilder zeigen eine klare Zweiteilung: Die Dienstleistungsfunktion hinsichtlich der Transparenz durch betriebswirtschaftliche Zahlen scheint im Wesentlichen klar zu sein. Hier wird nur die Gefahr gesehen, dass sich Controller zu stark in der Zahlenwelt verschanzen. Die Ergänzung und Begrenzung der Manager durch Controller („Counterpartfunktion“) dagegen ist offensichtlich in erheblichem Maße erklärungsbedürftig: Controller sind kompetenzmäßig Managern nicht gleichgestellt, sollen aber diese dennoch dazu bringen, anders zu handeln. Grund sind Könnens- und Wollensdefizite: Manager können sich zum einen aus Kompetenz- und/oder Zeitproblemen nicht ausreichend mit betriebswirtschaftlichen Aspekten beschäftigen. Manager handeln zum anderen potenziell opportunistisch.

Akzeptiert man diese grundsätzlichen Eigenschaften von Managern, ist es aber inkonsequent, wenn man sie für die Zahlenwelt gänzlich ausblendet. Hier herrscht bei Controllern immer noch ein "menschenloses" Weltbild vor, das sich in zwei Kernsätzen zusarmmenfassen lässt: (1) Zahlen lügen nicht (sie sind per se objektiv). (2) Mehr Informationen sind besser als wenige. Controller bemühen sich, Transparenz zu schaffen, indem sie immer mehr Informationen produzieren. Komfortable IT-Unterstützung treibt diese Entwicklung. Wer sagt denn aber, dass Manager damit zum Wohl des Unternehmens besser umgehen können? Ist Controllern die Tatsache wirklich so fremd, dass sich clevere Manager aus der Vielzahl der Daten immer genau die heraussuchen, mit denen sie gut dastehen? Welcher Controller hat detaillierte Kenntnis darüber, was Manager genau mit den Zahlen machen? Wer weiß, wie die Manager die Zahlen interpretieren? Wer

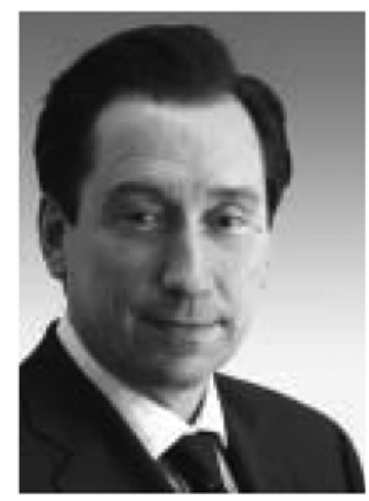

Prof. Dr. Jürgen Weber

ist sich sicher, dass die Manager die Zahlen wirklich verstehen? Wer kann angeben, wie viele Zahlen „seine“ Manager gleichzeitig richtig verarbeiten können? Welcher Controller hat sich schon einmal systematisch mit dem Phänomen des "Information Overload" beschäftigt?

Von Controllern wird derzeit viel Neues verlangt: Sie sollen sich mit den Gegebenheiten auf den Absatzmärkten auseinandersetzen (Kundenerfolg, Kundenwert), im Geschäftssystem nicht mehr nur auf Kosten achten, sondern auch mit Zeit- und Qualitätsgrößen richtig umgehen können (Reengineering, KVP), Kapitalmärkte in ihren Gesetzmäßigkeiten und Usancen verstehen (wertorientierte Steuerung). Der Wandel der Controllership sollte aber nicht auf die Übernahme neuer Aufgaben beschränkt bleiben. Controller müssen auch im angestammten Geschäft der Informationsversorgung des Managers umdenken. Das dazu passende Bild ist das eines Information-Gatekeepers des Managers. Weniger ist mehr!

Mit freundlichen Grüßen

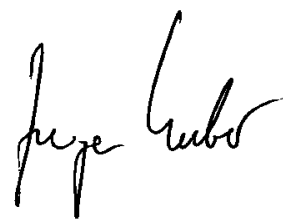

Ihr Jürgen Weber 